\title{
REGULAR BANACH ALGEBRAS WITH A COUNTABLE SPACE OF MAXIMAL REGULAR IDEALS
}

\author{
PAUL CIVIN AND BERTRAM YOOD
}

1. Introduction. In a recent paper [6] Wermer showed that, for the Banach algebra $C(D)$ of all complex continuous functions on the unit circle $D$ in the complex plane, the subalgebra $A$ of all functions in $C(D)$ analytic in $|z|<1$ is a maximal proper closed subalgebra. He also showed that if $L$ is a simple closed curve and $C(L)$ is the Banach algebra of all complex continuous functions on $L$ then $C(L)$ possesses a maximal proper closed subalgebra which separates points of $L$. Rudin [4] has shown that if $X$ is a compact Hausdorff space which contains a subset homeomorphic to the Cantor set then the Banach algebra $C(X)$ contains a maximal proper closed subalgebra which separates points of $X$. In the present note we show that if $X$ is a countable compact Hausdorff space then $C(X)$ contains no such maximal proper closed subalgebra. We obtain this result as a byproduct of the study of regular Banach algebras where we investigate a class of subalgebras which, in the case of $C(X)$, are the maximal proper closed subalgebras.

2. Definitions and preliminaries. Let $B$ be a complex commutative Banach algebra with space of maximal regular ideals $\mathfrak{M}$. Let $\pi: x \rightarrow x(M)$ be the Gelfand representation of $B$ as a subalgebra of $C(\mathfrak{M})$, the algebra of all complex continuous functions on $\mathfrak{M}$ which vanish at infinity. Where convenient we denote the function $x(M)$ also by $\hat{x}$. We call a subset $S$ of $B$ a separating family on $\mathfrak{M}$ if for each $M_{1}, M_{2}$ in $\mathfrak{M}, M_{1} \neq M_{2}$ there exists $x \in S$ such that $x\left(M_{1}\right) \neq x\left(M_{2}\right)$. A subalgebra $A$ of $B$ is called determining if $\pi(A)$ is dense in $\pi(B)$, otherwise $A$ is called nondetermining.

The notions of a maximal proper closed subalgebra and a maximal nondetermining subalgebra are related as follows.

2.1. Lemma. (a) $A$ maximal nondetermining subalgebra $A$ of $B$ is closed in $B$ and $\pi(A)$ is closed in $\pi(B)$.

(b) $A$ maximal proper closed subalgebra $A$ of $B$ which is nondetermining is a maximal nondetermining subalgebra.

(c) A maximal nondetermining subalgebra which is not a separating family on $\mathfrak{M}$ is a maximal proper closed subalgebra and is of the form $\left\{x \in B \mid x\left(M_{1}\right)=x\left(M_{2}\right)\right\}$ where $M_{1} \neq M_{2}$ in $\mathfrak{M}$.

Presented to the Society, February 25, 1956, under the title Maximal subalgebras in commutative Banach algebras; received by the editors January $20,1956$. 
Proof. (a) We show first that the closure of a nondetermining subalgebra $N$ is also nondetermining. For let $\|x\| \|=\sup |x(M)|$, $M \in \mathfrak{M}$. There exists $y \in B$ and $\epsilon>0$ such that $\|y-x\| \geqq \epsilon$ for all $x \in N$. Then it is easy to see that $\|y-z\| \| \geqq \epsilon$ for all $z \in \bar{N}$. Thus a maximal nondetermining subalgebra $A$ is closed. If $\pi(A)^{\circ}$ is the closure of $\pi(A)$ in $\pi(B)$ then $\pi^{-1}\left[\pi(A)^{c}\right]=A$ whence $\pi(A)$ is closed in $\pi(B)$.

(b) Let $A_{1}$ be the subalgebra generated by a maximal proper closed subalgebra $A$ and $x \notin A$ where $A$ is nondetermining. Since $A_{1}$ is dense in $B$ it follows that $\pi\left(A_{1}\right)$ is dense in $\pi(B)$.

(c) From the hypotheses there exist $M_{1}, M_{2} \in \mathfrak{M}, M_{1} \neq M_{2}$ such that $A \subset\left\{x \in B \mid x\left(M_{1}\right)=x\left(M_{2}\right)\right\}=A_{1}$, say. Since $A_{1}$ is the null-space of a linear functional on $B, A_{1}$ is a maximal proper closed subalgebra. As $A_{1}$ is nondetermining, $A=A_{1}$.

It is clear that any maximal nondetermining subalgebra of $B$ must contain the radical of $B$.

Following the usage in [2] and elsewhere, for a set $\mathfrak{F}$ in $\mathfrak{M}$ we define the kernel of $\mathfrak{F}, k(\mathfrak{F})=\cap M, M \in \mathfrak{F}$ and for an ideal $I$ in $B$, the hull of $I$ in $\mathfrak{M}$ as $h(I)=\{M \in \mathfrak{M} \mid M \supset I\} . B$ is called regular if the Gelfand topology for $\mathfrak{M}$ is the same as the hull-kernel topology for $\mathfrak{M}$ (see $[2$, p. 83] and also [5]). We discuss only regular Banach algebras $B$. Let $\mathfrak{F}$ be a closed set in $\mathfrak{M}$ and let $T$ be the natural homomorphism of $B$ onto $B / k(\mathfrak{F})$. As described in $\left[2\right.$, p. 76] $T$ defines a mapping $T^{*}$ of the space $\mathfrak{R}$ of maximal regular ideals of $B / k(\mathfrak{F})$ into $\mathfrak{M}$ by the rule $x\left(T^{*}(N)\right)=T(x)(N)$ for $x \in B, N \in \Re$. Moreover $T^{*}$ is a homeomorphism and, since $B$ is regular, $T^{*}(\mathfrak{N})=\mathfrak{F}$. Thus the Gelfand representation of $B / k(\mathfrak{F})$ may be thought of as the restriction of the functions in $\pi(B)$ to the set $\mathfrak{F}$ and we identify $\mathfrak{N}$ with $\mathfrak{F}$. For our purposes it is important to note that $B / k(\mathfrak{F})$ is regular $[7$, p. 164].

3. Regular Banach algebras with $\mathfrak{M}$ countable. Throughout this section $B$ denotes a complex commutative regular Banach algebra with space of maximal regular ideals $\mathfrak{M}$ where $\mathfrak{M}$ is countable. Let $\mathfrak{M}^{0}=\mathfrak{M}$ and for each ordinal $\alpha$ let $\mathfrak{M}^{\alpha}$ be the $\alpha$ th derived set of $\mathfrak{M}$. There is a first ordinal $\beta$ such that $\mathfrak{M}^{\beta}$ is void. For each $\alpha<\beta$ let $T_{\alpha}$ be the natural homomorphism of $B$ onto $B / k\left(\mathfrak{M}^{\alpha}\right)$.

3.1. Lemma. Let $\mathfrak{F}$ be a nonvoid compact subset of $\mathfrak{M}$. Then there is a last ordinal $\alpha$ such that $\mathfrak{M}^{\alpha} \cap \mathfrak{F}$ is not void.

PROOF. Since $\mathfrak{M}^{\beta} \cap \mathfrak{F}=\varnothing$ there exists a first ordinal $\mu$ such that $\mathfrak{M}^{\mu} \cap \mathfrak{F}=\varnothing$. Now $\mu$ cannot be a limit ordinal for otherwise

$$
\bigcap_{r<\mu} \mathfrak{M r} \cap \mathfrak{F}=\varnothing
$$


which is impossible since the $\mathfrak{M} \gamma \cap \mathfrak{F}$ form a decreasing set of nonvoid compact sets. Then there is an ordinal $\alpha$ such that $\mu=\alpha+1$.

3.2. Lemma. Let $L$ be a linear manifold in $B$ with the following property. For each $\alpha<\beta$ and each $M \in \mathbb{M}^{\alpha}$ which is an isolated point of $\mathfrak{M}^{\alpha}$ there exists $x \in L$ such that the function $x$ restricted to $\mathfrak{M}^{\alpha}$ is the characteristic function of $M$. Then the Gelfand representation of $L$ is dense in $C(\mathfrak{M})$.

Proof. Let $f(M) \in C(\mathfrak{M})$ and $\epsilon>0$. Let $\mathfrak{U}=\{M \in \mathfrak{M}|| f(m) \mid<\epsilon\}$. We show that there exists $g \in L$ such that $|g(M)-f(M)|<\epsilon, M \in \mathbb{M}$. We may suppose $f \neq 0$ and that $\epsilon$ is sufficiently small for the complement $\mathfrak{B}$ of $\mathfrak{U}$ to be nonvoid. Since $\mathfrak{B}$ is compact, by Lemma 3.1 there is a last ordinal $\alpha_{0}$ such that $\mathfrak{B} \cap \mathfrak{M}^{\alpha_{0}} \neq \varnothing$. Then $\mathfrak{B} \cap \mathfrak{M}^{\alpha_{0}}$ is a finite set, say $M_{1}, \cdots, M_{k}$, each point of which is an isolated point of $\mathfrak{M}^{\alpha}{ }^{\alpha}$. Let $h_{i} \in L$, where $h_{i}$ restricted to $\mathfrak{M}^{\alpha_{0}}$ is the characteristic function of $M_{i}, i=1, \cdots, k$. A linear combination $w_{0}$ of the $h_{i}$ may be chosen so that $w_{0}\left(M_{i}\right)=f\left(M_{i}\right), i=1, \cdots, k$. We have $w_{0}(M)=0$ for $M \in \mathfrak{M}^{\alpha_{0}} \cap \mathfrak{u}$. Thus $\left|w_{0}(M)-f(M)\right|<\epsilon, M \in \mathbb{M}^{\alpha_{0}}$.

If $\alpha_{0}=0$ or if $\left|w_{0}(M)-f(M)\right|<\epsilon$ for all $M \in \mathbb{M}$ we have the desired element. Otherwise let $\mathfrak{U}_{1}=\left\{M \in \mathfrak{M}|| w_{0}(M)-f(M) \mid<\epsilon\right\}$ and let $\mathfrak{B}_{1}$ be the complement of $\mathfrak{U}_{1}$. By Lemma 3.1 there is a last ordinal $\alpha_{1}$ such that $\mathfrak{B}_{1} \cap \mathfrak{M}^{\alpha_{1}} \neq \varnothing$. Clearly $\alpha_{1}<\alpha_{0}$. By the above procedure we may add a linear combination of elements of $L$ to $w_{0}$ obtaining $w_{1} \in L$ such that $\left|w_{1}(M)-f(M)\right|<\epsilon, M \in \mathfrak{M}^{\alpha_{1}}$. If $w_{1}$ is not the desired element and $\alpha_{1}>0$ we may repeat the procedure. Since a decreasing sequence of ordinals contains only a finite number of terms, at some finite stage we obtain an element $w_{n} \in L$ such that $\left|w_{n}(M)-f(M)\right|$ $<\epsilon, M \in \mathbb{M}$.

3.3. Theorem. (1) The subalgebra $B_{0}$ of $B$ consisting of all $x \in B$ such that $x$ has compact support has its Gelfand representation dense in $C(\mathfrak{M})$.

(2) An ideal $I$ of $B$ is contained in a regular maximal ideal of $B$ if and only if $I$ is nondetermining.

Proof. Consider $\mathfrak{M}^{\alpha}$ for $\alpha<\beta$ and $M_{0}$ an isolated point of $\mathfrak{M}^{\alpha}$. The Banach algebra $B / k\left(\mathfrak{M}^{\alpha}\right)$, being regular, contains an element $x$ whose Gelfand representation is the characteristic function of $M_{0}$ and $B$ contains an element $y$ where $T_{\alpha}(y)=x$. Let $\mathbb{B}$ be an open subset of $\mathfrak{M}$ with compact closure and $M_{0} \in\left(S\right.$. There exists $z \in B$ such that $z\left(M_{0}\right)$ $=1$ and $z(M)=0, M \notin \circlearrowleft$. Then for $w=y z, \widehat{w}$ restricted to $\mathfrak{M}^{\alpha}$ is the characteristic function of $M_{0}$ and has compact support. The conclusion (1) now follows from Lemma 3.2. 
Let $I$ be an ideal in $B$. If $I$ is contained in a maximal regular ideal then clearly $I$ is nondetermining. Suppose that $I$ is contained in no maximal regular ideal. Then $h(I)$ is void. By [2, p. 84, Theorem 24D] we see that $\pi\left(B_{0}\right) \subset \pi(I)$. By (1) of this theorem, $I$ is determining.

3.4. LemMA. Let $A$ be a maximal nondetermining subalgebra of $B$ which is not a maximal regular ideal of $B$. Suppose that $\mathfrak{M}$ is countable. Then either $\pi(A)$ contains the characteristic functions of all isolated points of $\mathfrak{M}$ or $A$ is not a separating family on $\mathfrak{M}$.

Proof. Suppose that $\pi(A)$ fails to contain the characteristic function $\delta$ of an isolated point $M_{0}$ of $\mathfrak{M}$. Let $\delta_{1} \in A, \pi\left(\delta_{1}\right)=\delta$. By Lemma $2.1, \delta$ is at a positive distance from $\pi(A)$ in $C(\mathfrak{M})$. Let $A_{1}$ be the algebra generated by $A$ and $\delta_{1}$ and let $L$ be the one-dimensional subspace of $C(\mathfrak{M})$ generated by $\delta$. Let the superscript $c$ denote closure in $C(\mathfrak{M})$. One easily verifies that $\pi\left(A_{1}\right)=\pi(A)+L$ is dense in $\pi(B)$ and that, since $\pi(A)^{c}+L$ is closed in $\pi(B)^{c}$, we have $\pi(A)^{c}+L=\pi(B)^{c}=C(\mathfrak{M})$ by Theorem 3.3. Let $\mathfrak{M}_{0}$ be the one-point compactification of $\mathfrak{M}$ and consider $C(\mathfrak{M})$ as a maximal ideal in $C\left(\mathfrak{M}_{0}\right)$. There exists a linear functional $x$ on $C\left(\mathfrak{M}_{0}\right)$ which vanishes on $\pi(A)^{c}$ and has the property that $x^{*}(\delta)=1$. Since $\pi(A)$ is closed in $\pi(B)$ by Lemma $2.1, x^{*-1}(0) \cap \pi(B)$ $=\pi(A)$. By the generalized Riesz representation theorem [1] there exists a completely additive regular set function $\mu$ defined for all Borel sets (and hence for all subsets) of $\mathfrak{M}_{0}$ such that for $f \in C\left(\mathfrak{M}_{0}\right)$,

$$
x^{*}(f)=\int_{\mathbb{M}_{0}} f(M) d \mu .
$$

Since $x^{*}(\delta)=1$ we have $\mu\left(\left\{M_{0}\right\}\right)=1$. It is impossible that $\mu(\{M\})=0$ for all $M \in \mathfrak{M}_{0}, M \neq M_{0}$. For otherwise since there exists $g \in \pi(A)$ such that $g\left(M_{0}\right) \neq 0$ we have $x^{*}(g)=g\left(M_{0}\right) \neq 0$ which is impossible.

Let $M_{i} \in \mathfrak{M}, M_{i} \neq M_{0}, i=1,2$ where $M_{1} \neq M_{2}$. We show that it is impossible to have $\mu\left(\left\{M_{1}\right\}\right) \neq 0$ and $\mu\left(\left\{M_{2}\right\}\right) \neq 0$. Suppose otherwise. Since $\mathfrak{M}_{0}$ is zero-dimensional there exist open and closed neighborhoods $\mathfrak{B}_{i}$ of $M_{i}, i=1,2$ which are disjoint and contain neither $M_{0}$ nor the point at infinity. It is clear that each $\mathfrak{B}_{i}$ is an open and closed compact subset of $\mathfrak{M}$. By Theorem 3.3 , the functions $\hat{x}, x \in B$, restricted to $\mathfrak{B}_{1}$ form a dense set in $C\left(\mathfrak{B}_{1}\right)$. Let $\mu_{1}$ be the set function $\mu$ restricted to subsets of $\mathfrak{B}_{1}$. It is clear that $\mu_{1}$ is a completely additive regular set function on $\mathfrak{B}_{1}$. Therefore if

$$
\int_{\mathfrak{B}_{1}} f(M) d \mu_{1}=0
$$

for all $f \in \pi(B)$ we see from $\left[1\right.$, p. 39] that (1) holds for all $f \in C\left(\mathfrak{B}_{1}\right)$ 
and that $\mu_{1}(E)=0$ for all Borel sets in $\mathfrak{B}_{1}$. This implies that $\mu\left(\left\{M_{1}\right\}\right)$ $=0$ which is contrary to our assumptions. Hence there exists $x_{1} \in B$ such that

$$
\int_{\mathfrak{B}_{1}} x_{1}(M) d \mu_{1}=-1 .
$$

By regularity there exists $y_{1} \in B$ such that $y_{1}(M)=0, M \in \mathfrak{B}_{1}$ and $y_{1}\left(M_{0}\right)=1-x_{1}\left(M_{0}\right)$. Set $u_{1}=x_{1}+y_{1}$. By a theorem of Silov (see [2, p. 84] and $\left[5\right.$, p. 37]) there exists $v_{1} \in B$ such that $v(M)=1, M \in \mathfrak{B}_{1}$ $\cup\left\{M_{0}\right\}$ and $v_{1}(M)=0$ elsewhere. Set $w_{1}=u_{1} v_{1}$. Then $w_{1}\left(M_{0}\right)=1$, $w_{1}(M)=x_{1}(M)$ for $M \in \mathfrak{B}_{1}$ and $w_{1}(M)=0$ elsewhere. Therefore

$$
x^{*}\left(w_{1}\right)=w_{1}\left(M_{0}\right)+\int_{\mathfrak{B}_{1}} w_{1}(M) d \mu_{1}=0 .
$$

Hence $w_{1} \in \pi(A)$. In the same way we find $w_{2} \in \pi(A)$ where $w_{2}\left(M_{0}\right)=1$ and $w_{2}(M)=0, M \notin \mathfrak{B}_{2} \cup\left\{M_{0}\right\}$. But $\widehat{w}_{1} \widehat{w}_{2}=\delta$ and $\widehat{w}_{1} \widehat{w}_{2} \in \pi(A)$. This contradicts the hypothesis that $\delta \notin \pi(A)$.

Consequently there exists exactly on $M_{1} \in \mathfrak{M}, M_{1} \neq M_{0}$ such that $\mu\left(\left\{M_{1}\right\}\right)=a \neq 0$. For $f \in \pi(B), \quad x^{*}(f)=f\left(M_{0}\right)+a f\left(M_{1}\right)$. Consider $g \in \pi(A)$. If $g\left(M_{1}\right)=0$ then $g\left(M_{0}\right)=0$. Suppose that $g\left(M_{1}\right)=-1$. Then $g\left(M_{0}\right)-a=0$. Since $g^{2} \in \pi(A),\left[g\left(M_{0}\right)\right]^{2}+a=0$. Then $a=-1$. This shows that $g\left(M_{0}\right)=g\left(M_{1}\right)$ here also. Therefore $A$ is not a separating family.

3.5. TheOREM. The maximal proper closed subalgebras of $B$ which are nondetermining are the maximal regular ideals of $B$ and the sets of the form $\left\{x \in B \mid x\left(M_{1}\right)=x\left(M_{2}\right), M_{i} \in \mathfrak{M}, i=1,2, M_{1} \neq M_{2}\right\}$.

Proof. In view of Lemma 2.1 it is sufficient to show that a maximal nondetermining subalgebra $A$ of $B$ which is not a maximal regular ideal is not a separating family on $\mathfrak{M}$. Suppose otherwise.

We show, by transfinite induction, that $T_{\alpha}(A)$, for each $\alpha<\beta$, is a maximal nondetermining subalgebra of $B / k\left(\mathfrak{M}^{\alpha}\right)$ which is a separating family on $\mathfrak{M}^{\alpha}$. The conclusion is trivial for $\alpha=0$. Suppose that the assertion is true for all ordinals $\gamma, \gamma<\alpha<\beta$. We establish first that $T_{\alpha}(A)$ is a nondetermining subalgebra of $B / k\left(\mathfrak{M}^{\alpha}\right)$. For suppose otherwise. Let $f(M) \in \pi(B)$ and $\epsilon>0$. We shall obtain a contradiction by demonstrating that there exists $g \in A$ such that $|g(M)-f(M)|<\epsilon$, $M \in \mathfrak{M}$. There exists $h \in A$ such that $|h(M)-f(M)|<\epsilon, M \in \mathfrak{M}^{\alpha}$. Let $\mathfrak{U}=\{M \in M|| h(M)-f(M) \mid<\epsilon\}$. The complement $\mathfrak{B}$ of $\mathfrak{U}$ is compact. By Lemma 3.1 there is a last ordinal $\alpha_{0}$ such that $\mathfrak{M} \alpha_{0} \cap \mathfrak{B} \neq \varnothing$. Clearly $\alpha_{0}<\alpha$. By Lemma 3.4 (applied to the regular Banach algebra $B / k\left(\mathfrak{M}^{\alpha_{0}}\right)$ ) we see that for each isolated point $M_{0}$ of $\mathfrak{M}^{\alpha_{0}}, A$ contains 
an element $x$ such that when $x(M)$ is restricted to $\mathfrak{M}^{a_{0}}$ the characteristic function of $M_{0}$ on $\mathfrak{M}^{\alpha_{0}}$ is obtained. Arguing as in Lemma 3.2, we see that there exists $w_{1} \in A$ such that $\left|w_{1}(M)-f(M)\right|<\epsilon, M \in \mathfrak{M}^{\alpha_{0}}$. By repeated use of Lemma 3.4 and by the arguments of Lemma 3.2 we obtain the desired $g \in A$.

We also observe that $T_{\alpha}(A)$ is a maximal nondetermining subalgebra of $B / k\left(\mathfrak{M}^{\alpha}\right)$. For if $Q$ is a nondetermining subalgebra which properly contains $T_{\alpha}(A)$ then $T_{\alpha}^{-1}(Q)$ is a nondetermining subalgebra of $B$ which properly contains $A$ (see $\S 2$ ). It is clear that $T_{\alpha}(A)$ is a separating family on $\mathfrak{M}^{\alpha}$. Thus the induction is complete.

If $\alpha<\beta$ and $M_{0}$ is an isolated point of $\mathfrak{M}^{\alpha}$, by the above and Lemma 3.4 $A$ possesses an element $x$ such that $\hat{x}$, restricted to $\mathfrak{M}^{\alpha}$, is the characteristic function of $M_{0}$. Hence by Lemma 3.2, $A$ is determining. This is a contradiction and the proof is complete.

3.6. CoRollary. Let $B$ be a complex commutative $B^{*}$-algebra with a countable space $\mathfrak{M}$ of maximal regular ideals. Then the maximal proper closed subalgebras of $B$ are the maximal regular ideals of $B$ and the sets of the form $\left\{x \in B \mid x\left(M_{1}\right)=x\left(M_{2}\right), M_{i} \in \mathfrak{M}, i=1,2, M_{1} \neq M_{2}\right\}$.

Proof. This follows from Theorem 3.5 since in this case it is clear that the maximal proper closed subalgebras are the maximal nondetermining subalgebras.

Let $G$ be any locally compact abelian group whose character group $\hat{G}$ is countable, e.g. $G$ a generalized toroidal group $[3$, p. 142$]$. Let $B$ be the group algebra of $G$. Then $B$ satisfies the hypotheses of Theorems 3.3 and 3.5. Thus the maximal nondetermining subalgebras of $B$ are completely described by the above results.

\section{BibliogRAPHY}

1. N. Dunford, Spectral theory in abstract spaces and Banach algebras, Proceedings of the Symposium on Spectral Theory and Differential Problems, Stillwater, Okla., 1951.

2. L. H. Loomis, An introduction to abstract harmonic analysis, New York, Van Nostrand, 1953.

3. L. Pontrjagin, Topological groups, Princeton University Press, 1939.

4. W. Rudin, Maximal subalgebras in spaces of continuous functions, Bull. Amer. Math. Soc. Abstract 61-4-486.

5. G. Silov, On regular normal rings, Traveux de l'Institut Mathématique Stekloff vol. 21 (1947) (Russian, English summary).

6. J. Wermer, On algebras of continuous functions, Proc. Amer. Math. Soc. vol. 4 (1953) pp. 866-868.

7. B. Yood, Topological properties of homeomorphisms between Banach algebras, Amer. J. Math. vol. 76 (1954) pp. 155-167.

UNIVERSITY OF OREGON 